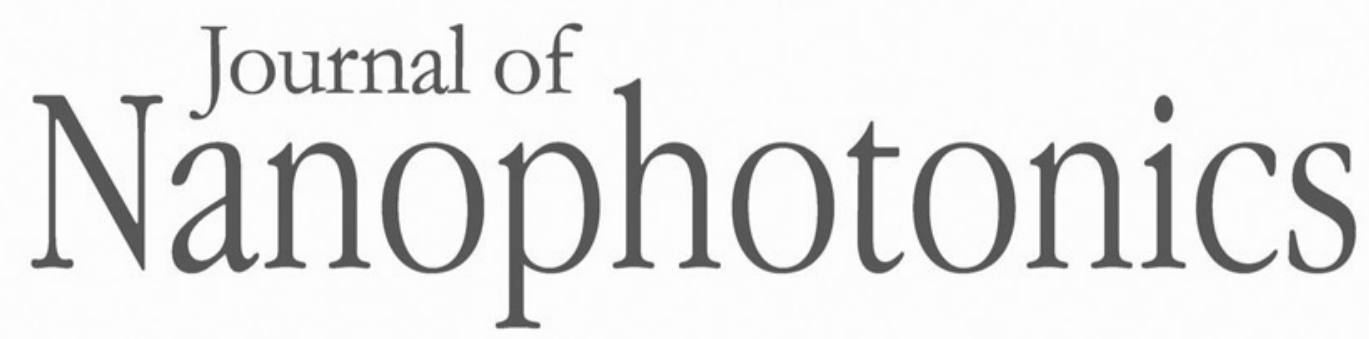

Nanophotonics.SPIEDigitalLibrary.org

\title{
Special Section Guest Editorial: Optical Manipulation and Structured Materials
}

\author{
Takashige Omatsu \\ Masaaki Ashida \\ Yoshihiko Arita
}




\title{
Special Section Guest Editorial: Optical Manipulation and Structured Materials
}

\author{
Takashige Omatsu, ${ }^{\text {a,b }}$ Masaaki Ashida, ${ }^{c}$ and Yoshihiko Arita ${ }^{\text {b,d }}$ \\ ${ }^{\mathrm{a}}$ Chiba University, Graduate School of Engineering, 1-33 Yayoi-cho, Inage-ku, \\ Chiba-shi 263-0022, Japan \\ ${ }^{\mathrm{b}}$ Chiba University, Molecular Chirality Research Center, 1-33 Yayoi-cho, Inage-ku, \\ Chiba-shi 263-0022, Japan \\ 'Osaka University, Graduate School of Engineering Science, 1-3 Machikaneyama, \\ Toyonaka-shi 560-8531 Japan \\ ${ }^{\mathrm{d}}$ University of St. Andrews, SUPA, School of Physics \& Astronomy, North Haugh, \\ Fife, KY16 9SS, United Kingdom
}

Conventional optical tweezers rely on the field gradients near the diffraction limited focus of a laser beam. Dielectric particles with a dimension ranging from hundreds of nanometers to tens of micrometers can be stably trapped by the gradient force at the beam focus. However, this optical technique does not always allow efficient trapping and manipulation of particles at the nanoscale because the gradient force scales with the volume of the particle. It is thus highly desirable to develop novel trapping geometries and materials that can significantly reinforce the interaction between optical fields and trapping materials at this length scale. Recently, near-field optical trapping techniques (e.g., using metamaterials and plasmonic and photonic crystals) have enabled the optical manipulation of nanoparticles at dimensions beyond the diffraction limit and have made it possible to trap single biomolecules. Furthermore, structured light fields (e.g., optical vortices, vector beams, and nondiffraction beams) have played an important role in understanding more complex light-matter interactions, including the transfer of spin and orbital angular momentum of light to matter. This special section comprises six contributed articles, which should enable us to further understand the interplay between structured light fields and structured materials on a subwavelength scale for advanced optical manipulation.

Mansuripur theoretically investigated the exchange of linear and angular momentum from an electromagnetic wave-packet to a small spherical particle and revealed different optomechanical responses when the particle absorbed energy from the incident light. Setoura et al. applied fluorescence correlation spectroscopy to monitor the temperature around a trapped particle with numerical simulations. Leménager et al. investigated the trapping characteristics of a range of nanoparticles including spherical YAG:Ce $\mathrm{Ce}^{3+}$ and $\mathrm{NaYF} 4 \mathrm{Er}^{3+}, \mathrm{Yb}^{3+}$ nanorods in optical fiber tweezers. Sun et al. reported on a surface plasmon resonance imaging technique using a three-dimensional plasmonic microwell array to enhance the electromagnetic field response. Anbardan et al. introduced a technique to synchronize two cavity solitons in a driven vertical cavity surface emitting laser above threshold. Furthermore, structured light-matter interactions enabled the fabrication of novel material structures. Minowa et al. fabricated cadmium selenide quantum dots using a pulsed laser-ablation scheme in superfluid helium.

Finally, we would like to thank all authors and reviewers, as well as the editorial and publication team of Journal of Nanophotonics, for their contributions to the Special Section on Optical Manipulation and Structured Materials.

Takashige Omatsu is a professor at Chiba University in Japan. He received his $\mathrm{PhD}$ degree in physics from the University of Tokyo in 1992. His current research interests include laser physics, structured light, and optical materials processing and manipulation. He is the head of the Molecular Chirality Research Center, Chiba University, and the editor-in-chief of OSA Continuum (The Optical Society). He is a fellow of OSA, JSAP, and SPIE.

Masaaki Ashida is a professor at Osaka University in Japan. He received his $\mathrm{PhD}$ degree in physics from Kyoto University in 1994. His current research interests include optical manipulation of material properties, advanced photonics, and condensed-matter systems. He is a member of OSA, JPSJ, and JSAP. 
Yoshihiko Arita is a research fellow at the University of St. Andrews in the UK and a research associate professor at Chiba University in Japan. He received his DPhil degree in atomic and laser physics from the University of Oxford in 2008. His current research spans a wide range of optical manipulation studies, including trapping in vacuum-levitated optomechanics, microrheology, and materials processing. He is a member of OSA and SPIE. 